\title{
Low loss poly(methyl methacrylate) useful in polymer optical fibers technology
}

\author{
Pawel Mergo*, Malgorzata Gil, Krzysztof Skorupski, Jacek Klimek, Grzegorz Wójcik, Janusz Pędzisz, \\ Jaroslaw Kopec, Krzysztof Poruraj, Lidia Czyzewska, Aleksander Walewski, Andrzej Gorgol \\ Laboratory of Optical Fibre Technology, Maria Curie-Sklodowska University, \\ Pl. Sklodowskiej 3, 20-031 Lublin, Poland
}

Received December 27, 2013; revised December 29, 2013; accepted December 30, 2013; published December 31 , 2013

\begin{abstract}
The paper presents comparison of thermal and optical properties of the typical commercial available and manufactured in our laboratory poly(methyl metacrylate) polymer useful in polymer optical fibers technology. The thermal studies were made by thermogravimeter connected on-line with FT-IR and QMS spectrometer, the optical properties were characterized by spectral attenuation measurements. It was found that polymer obtained in our laboratory is characterized by higher temperature (about $100^{\circ} \mathrm{C}$ ) of beginning mass loss occurred and lower attenuation then commercial available polymer.
\end{abstract}

Microstructured optical fibers (MOFs) provide extra degrees of freedom in manipulating the optical properties of light, such as dispersion, nonlinearity, and birefringence [1-5]. Therefore they have attracted increased interest over the last two decades. Due to specific material properties, the technology of microstructured polymer optical fibers (mPOF) has been intensively developed in recent years. Polymer fibers may show better parameters than fibers made of silica glass in a variety of applications. One of the advantages is biological compatibility, which opens the possibility for medical applications. Moreover polymers have higher flexibility than silica glass, therefore can withstand much higher strains, which enables applications of polymer fibers as elongation sensors in a strain range unattainable for silica fibers [6]. In order to obtain a polymer optical fiber with the proper optical characteristics, it is necessary to prepare specific preform from which optical fibers are drawn or extruded. Now polymer optical fibers are mainly manufactured from poly(methyl methacrylate) PMMA (linear polymer), produced by the polymerization of methyl methacrylate. Unfortunately the main disadvantage of "PMMA optical fibers" is their very high losses, in comparison with "silica fibers", mainly caused by $\mathrm{C}-\mathrm{H}$ bonds and impurities. In this letter we present the low loss poly(methyl metacrylate) polymer manufactured in our Laboratory. The manufactured from our polymer $\mathrm{mPOF}$ was characterized by significant decrease of the losses.

\section{*E-mail: pawel.mergo@poczta.umcs.lublin.pl}

A sample of "our PMMA" was prepared from methyl methacrylate (ALDRICH). In polymer technology two methods of polymerization are used - thermal and photopolimerization. The chemical reaction, essentially, takes place in the same way, except the source of initiation. Due to the crosslinking of the polymer during photopolymerization, this technique is not used in the polymer optical fibers technology. A very important issue in the production of materials for optical applications is their purity. Even a small amount of impurities can significantly decrease the transmission properties of the final product such as an optical fiber. Therefore, MMA was purified before starting the process of polymerization. The purification is performed by distillation process in under pressure conditions getting rid of impurities as well as undesirable inhibitor, which is added to prevent the initial process of prepolymerization of methyl methacrylate. The initiator benzoyl peroxide (ALDRICH), which was also purified before the use, and chain transfer agent thioglycolic acid (98\% ALDRICH) were used in thermal free radical polymerization of examined samples. Sample was prepared as described in [7].

In order to demonstrate the advantageous properties of the PMMA obtained in our Laboratory compared to the commercially available PMMA, a comparison of thermogravimetric (TG), spectroscopic (MS and FT-IR) and optical analysis both polymers was made. The chemical composition of the "our" sample consisted of: methyl methacrylate, benzoyl peroxide $(0,4 \% \mathrm{w} / \mathrm{w})$ and thioglycolic acid $(0,8 \% \mathrm{w} / \mathrm{w})$ which were used as an initiator and as a chain transfer agent respectively.

Thermal analysis was carried out on a STA 449 Jupiter F1, Netzsch (Selb, Germany) under the following operational conditions: heating rate $10^{0} \mathrm{C} \mathrm{min}^{-1}$, a dynamic atmosphere of helium $\left(40 \mathrm{~mL} \mathrm{~min}^{-1}\right)$ in the temperature range of $40^{\circ} \mathrm{C}-600^{\circ} \mathrm{C}$, sample weight of about $10 \mathrm{mg}$, sensor thermocouple type S TG-DSC. The identification of a gas composition coming out during depolymerization and decomposition process were detected and analyzed by quadrupole mass spectrometer QMS 403C Aëolos 
(Germany) as well as FT-IR spectrometer Brucker Tensor 27 TGA 585 (Germany) coupling on-line to STA instrument. The QMS was operated with an electron impact ionizer with energy $70 \mathrm{eV}$. The FT-IR spectrometer with IR cell maintained at $200^{\circ} \mathrm{C}$ was connected on-line to STA instrument by transfer line evolved gases from TGA to the FT-IR with diameter of $2 \mathrm{~mm}$ heated to $200^{\circ} \mathrm{C}$. The FT-IR spectra were recorded in the spectral range of $600-4000 \mathrm{~cm}^{-1}$ with 16 scans per spectrum at a resolution of $4 \mathrm{~cm}^{-1}$. The optical properties of both "our" and commercial polymers were characterized by spectral attenuation measurements.

Both samples were subjected to thermogravimetric analysis, based on which it was possible to analyze the initial weight loss temperature and decomposition temperatures. The analysis was performed in two ranges of weight loss: $0.01 \% \mathrm{w} / \mathrm{w}-0.05 \% \mathrm{w} / \mathrm{w}$ (Table 1) and $1 \% \mathrm{w} / \mathrm{w}-5 \% \mathrm{w} / \mathrm{w}$ (Table 2 ).

Table 1. Determination of weight loss temperature from $0,01 \mathrm{w} / \mathrm{w} \%$ to $0,05 \mathrm{w} / \mathrm{w} \%$.

\begin{tabular}{|c|c|c|c|c|c|}
\hline \multirow{2}{*}{ Sample } & \multicolumn{5}{|c|}{ Weight loss [\%w/w] and temperature at which it occurs $\left[{ }^{\circ} \mathrm{C}\right]$} \\
\cline { 2 - 6 } & 0.01 & 0.02 & 0.03 & 0.04 & 0.05 \\
\hline $\begin{array}{c}\text { "Our" } \\
\text { PMMA }\end{array}$ & 284.5 & 284.9 & 285.3 & 285.7 & 286 \\
\hline $\begin{array}{c}\text { Comm. } \\
\text { PMMA }\end{array}$ & 110 & 113.1 & 115.1 & 118.3 & 120 \\
\hline
\end{tabular}

The data presented in Table 1, clearly show that "our" PMMA has a higher strength and thermal stability. It should be clearly pointed out that the temperature at which the first weight loss is observed $(0.01 \% \mathrm{w} / \mathrm{w})$ is $285^{\circ} \mathrm{C}$ for the sample obtained in our Laboratory, and only $110^{\circ} \mathrm{C}$ for commercial PMMA. It should also be noted that samples exhibited a significant difference in the decomposition temperature (Table 2).

Table 2. Determination of weight loss temperature from $1 \mathrm{w} / \mathrm{w} \%$ to $5 \mathrm{w} / \mathrm{w} \%$.

\begin{tabular}{|c|c|c|c|c|c|c|}
\hline \multirow{2}{*}{ Sample } & \multicolumn{5}{|c|}{ Weight loss [\%w/w] and temperature at which it occurs $\left[{ }^{0} \mathrm{C}\right]$} \\
\cline { 2 - 7 } & 1 & 2 & 3 & 4 & 5 & $\mathrm{~T}_{\mathrm{I}}^{*}$ \\
\hline $\begin{array}{c}\text { Our } \\
\text { PMMA }\end{array}$ & 310 & 324 & 332 & 338 & 342 & $0.01-285$ \\
\hline $\begin{array}{c}\text { Comm. } \\
\text { PMMA }\end{array}$ & 225 & 300 & 313 & 320 & 325 & $1.27-268$ \\
\hline \\
Initial decomposition weight loss/temperature
\end{tabular}

Commercially available PMMA begins its proper decomposition at $268^{\circ} \mathrm{C}$, it is not a low temperature, but it should be noted that at this temperature for this sample $1,27 \%$ w/w of weight loss has already been registered.
Whereas the "our polymer" in the decomposition temperature exhibits only a $0,01 \% \mathrm{w} / \mathrm{w}$ of weight loss (Figure 1).

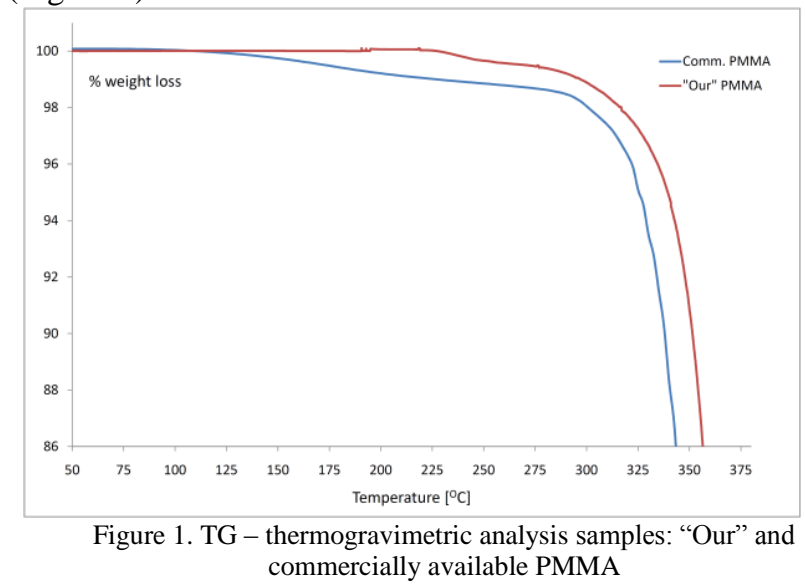

These temperature values are important parameters which determine the processing properties of the tested materials. The beginning of weight loss and its increase as the temperature rises will provide the thermal stability of the analyzed materials, whereas about the thermal resistance, the initial decomposition temperature. It is worth also take into account the temperature at which the polymer optical fibers are drawn $\left(250^{\circ} \mathrm{C}-270^{\circ} \mathrm{C}\right)$. On the basis of this information it can be concluded that sample "our PMMA" has a much better thermal properties and thus process ability as compared to the commercially available PMMA.

On the basis of MS and IR spectra, the analyses of gases evolved during the temperature increase of the samples were made. In the case of technical PMMA it was found that the weight loss, which was observed on TG curve (Figure 1), results from the insufficient polymerization degree. This observation was made on the basis of the presence (from $110^{\circ} \mathrm{C}$ ) in evolved gases ions: 41 and 69 resulting from the fragmentation of MMA molecules, containing in their structure double bonds. It should beadded that the presence of ions: 27 and 29 - an aldehyde and vinyl group derived from MMA molecules (from $148^{\circ} \mathrm{C}$ ) was also observed. IR spectra, in the analyzed temperature range, registered a small presence of water (the spectral range $3900 \mathrm{~cm}^{-1}-3000 \mathrm{~cm}^{-1}$ ) and bands corresponding to the stretching vibrations of $\mathrm{C}-\mathrm{O}$ methyl ester and the rotational vibration of the methyl ester $-\mathrm{CH}_{3}$ (spectral range $1300 \mathrm{~cm}^{-1}-1050 \mathrm{~cm}^{-1}$, from $150^{\circ} \mathrm{C}$ ). The same analysis was performed for the sample obtained in our Laboratory. On the basis of MS spectra the presence of discussed ions were not observed. Only ion 41 appears in a small amount at a temperature of $127^{\circ} \mathrm{C}$. While IR spectra registered in the spectral range $1300 \mathrm{~cm}^{-1}-1050 \mathrm{~cm}^{-1}$ absorption increase in the temperature from $180^{\circ} \mathrm{C}$ to $300^{\circ} \mathrm{C}$. 
The rods about external diameter $1 \mathrm{~mm}$ were drawn on the polymer optical fiber drawing tower from both ("our" and commercially available) PMMA. The spectral attenuations were measured by the standard cut-back method. For protection against penetration of external light rods were placed in special black box. Figure 2 presents spectral attenuation of the "our" PMMA. Figure 3 presents spectral attenuation of the commercially available PMMA.

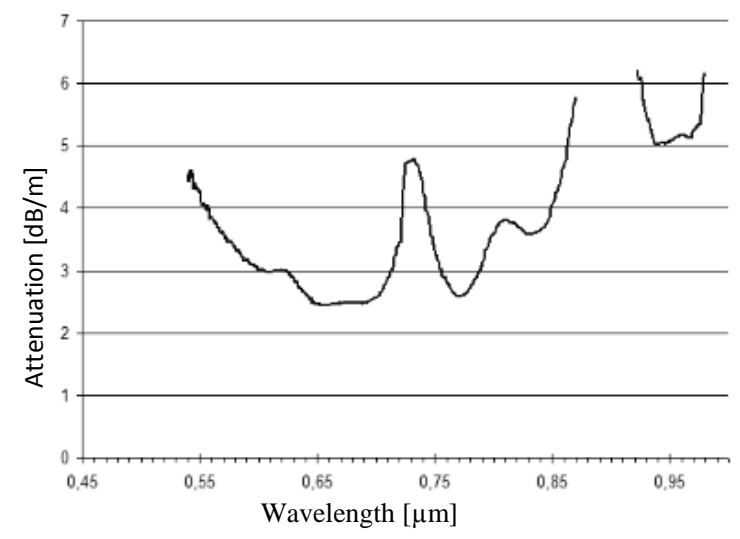

Figure 2. Spectral attenuation of "our" PMMA

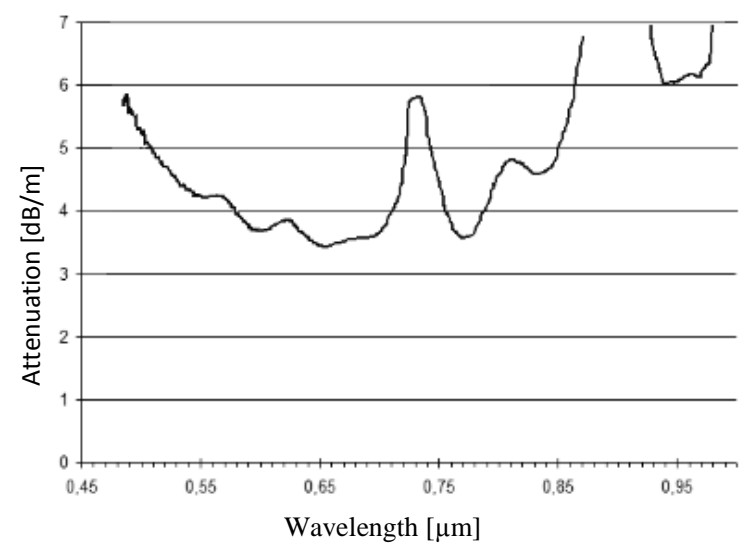

Figure 3. Spectral attenuation of commercially available PMMA
In this study, a comparison of two polymers used in optical fibers technology, was made. On the basis of presented studies it was concluded that PMMA obtained in our laboratory has a higher strength and thermal stability and that means better process ability as compared to the commercially available PMMA. Optical studies confirm presented conclusions. Attenuation of "our PMMA" is lower than that measured for the commercially available PMMA.

This work was supported by Wrocław Research Center EIT+ Ltd in the frame of the NanoMat project Application of Nanotechnology in Advanced Materials, within the European Funds for Regional Development, POIG, Subaction 1.1.2.

\section{References}

[1] P.St.J. Russell, J.C. Knight, T.A. Birks, B.J. Mangan, W.J. Wadsworth, Science 299, 358-362 (2003),

[2] J.C. Knight, Nature 424, 847-851 (2003),

[3] A. Ortigosa-Blanch, J.C. Knight, W.J. Wadsworth, J. Arriaga, B.J. Mangan, T.A. Birks, and P.S.J. Russell, Opt. Lett. 25, 1325-1327 (2000),

[4] G. Farrell, Phot. Lett. Poland. 4, 124 (2012),

[5] A. Umińska, M. Grabka, S. Pustelny, R. Buczyński, I. Kujawa, W. Gawlik, Phot. Lett. Poland. 4, 140-142 (2012),

[6] D. Webb and K. Kalli, "Polymer fiber Bragg gratings", in Fiber Bragg Grating Sensors: Recent Advancements, Industrial Applications and Market Exploitation, A. Cusano, A. Cutolo, and J. Albert, Eds. Sharjah (Bentham Science Publishers 2011), pp. 292-312,

[7] Mergo P., Gil M., Podkościelny W., Worzakowska M., Adsorption 19. 851-859 (2013). 